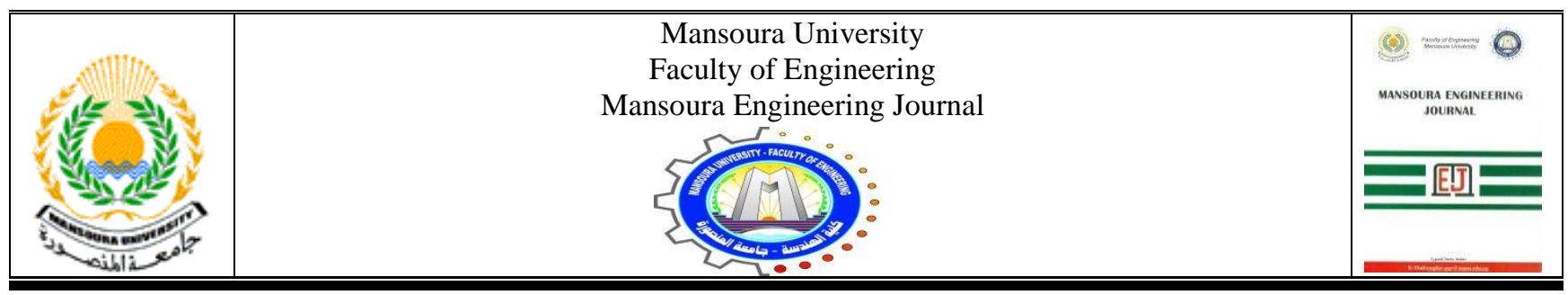

\title{
The Impact of Advanced Construction Technology on Refugee Shelters
}

\author{
Asmaa Ahmed Abd El-Hay El-Taweel and Methat A. Samra
}

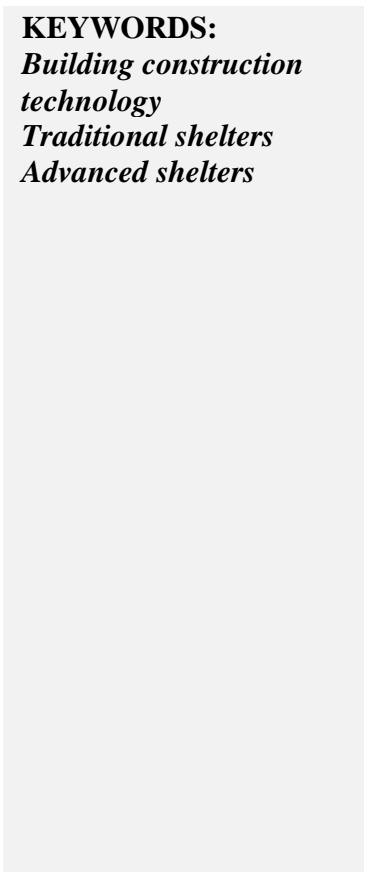

\begin{abstract}
Many refugees are forced into light camps that make them vulnerable to physical and emotional dangers, and thus suffer a complete break in the social, economic and material aspects of life. The emergency camps are planned, randomly designed and intended only to provide protection from natural elements (floods - earthquakes ...) and industrial elements (wars .) which are usually built in large open areas made of simple tent structures, always temporary housing ( The camp) does not include living and sleeping spaces, as well as areas for food preparation, personal hygiene and privacy, but rather one space.

Therefore, the research paper aims to monitor the evolution of building technology variables for light installations at the global level, with the aim of reaching their economic potential for application and exploitation in our society. In order to benefit from these developments in making refugee camps quickly and with high accuracy and environmentally friendly materials, and to identify the efficiency of assistive technologies for the work of light installations, especially for refugee camps, quickly and accurately, and their ability to deal with a complex creative process.

The paper will deal with assessing the efficiency of advanced technologies compared to the traditional shelters commonly used in refugee camps, by developing technologies to create a (camp) model that helps in studying the cost, technical specifications, life span and delivery time for an advanced shelter. After comparison, it was found that the advanced shelter achieved lower construction cost, higher durability, and could be implemented in less time than traditional shelters.
\end{abstract}

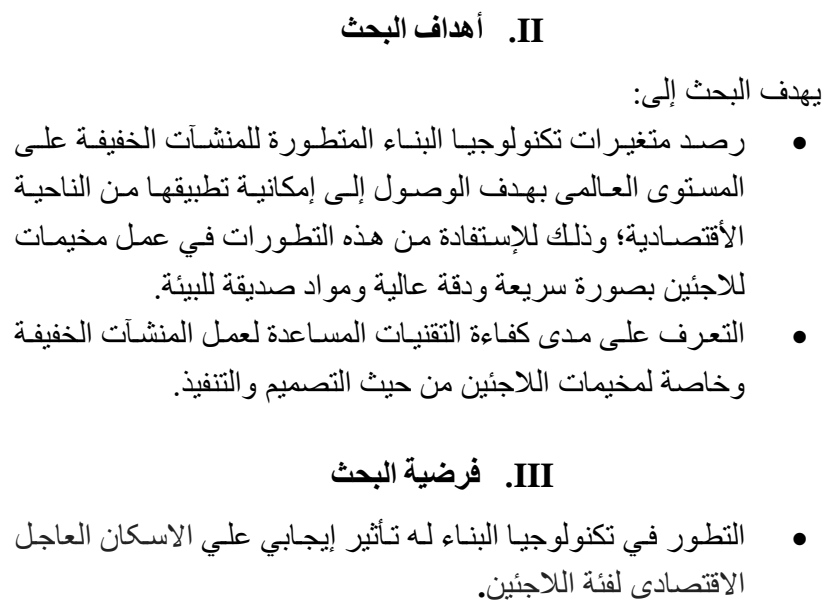

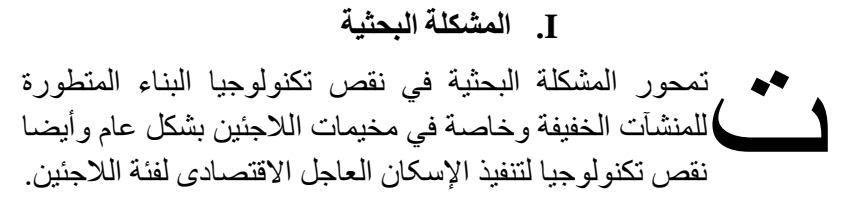

Received: (20 September, 2020) - Revised: (14 November, 2020) Accepted: (22 November, 2020)

Corresponding Author: Eng. Asmaa Ahmed Abd El-Hay El-Taweel (Demonstrator, Architectural Dept, Delta High Institute for Engineering and Technology, Mansoura). (e-mail: asmaaeltawil44@gmail.com).

Dr. Methat A. Samra (Lecturer, Architectural Dept, Mansoura University (e-mail: medhatsat2005@hotmail.com). 


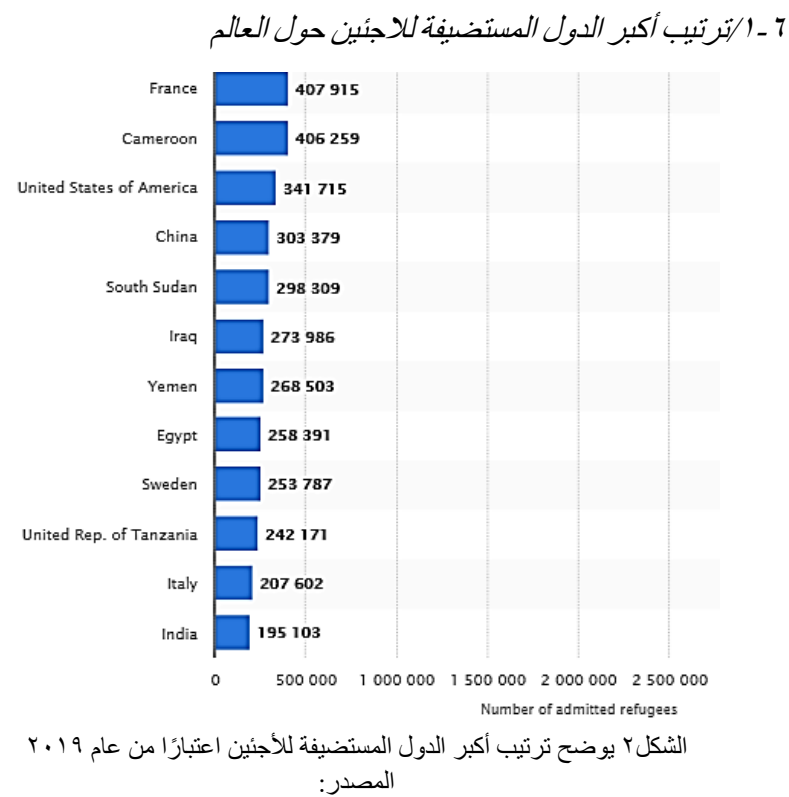

: https://www.statista.com/statistics/263423/major-refugeehosting-countries-worldwide/(date5/9/2020 7:28pm)

7 ـ إب / المشاكل التي تو/جه اللاجئين بالمخبيات

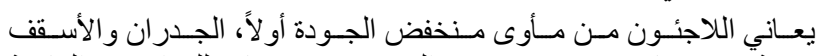

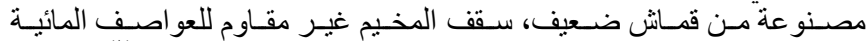

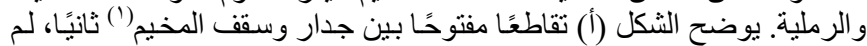

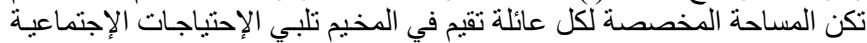

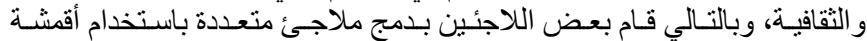

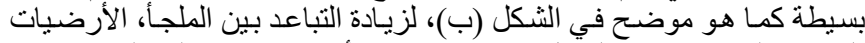

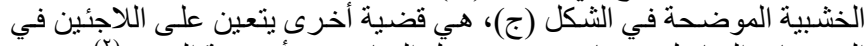

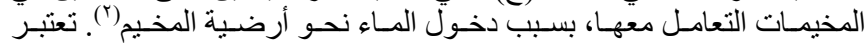

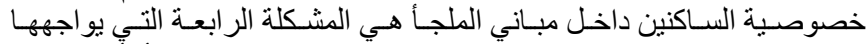

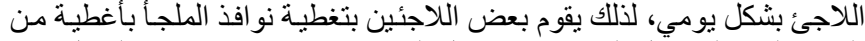

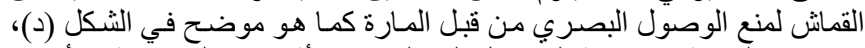

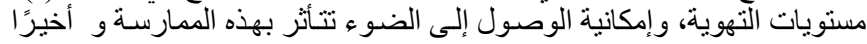

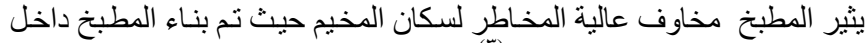
مو اد إيو اء عالية القابلية للاشتعال. (r)
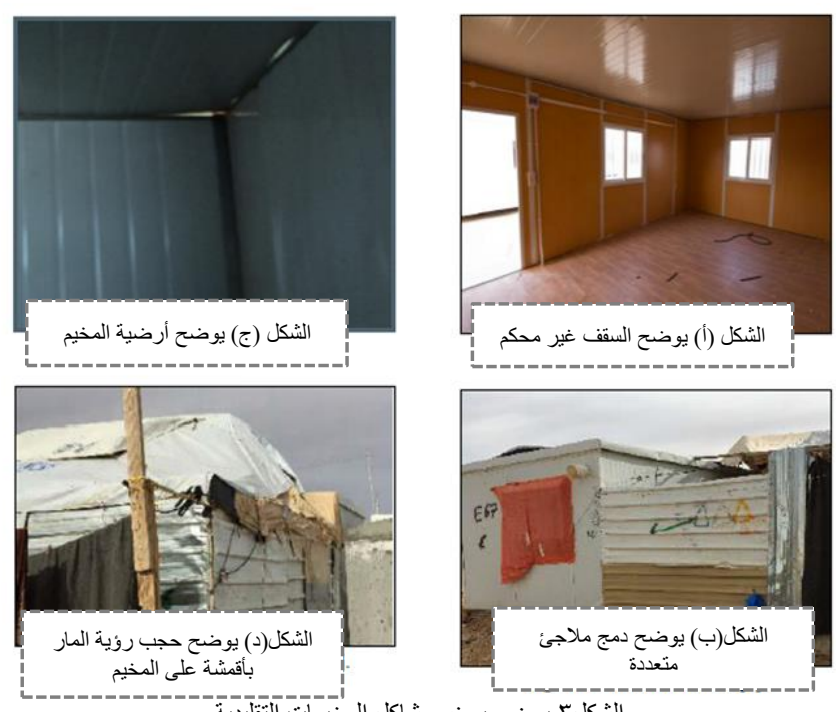

$$
\text { المصن مشاكل المر: }
$$

Mohanad Akeila, Kelvin Kuok King Kuok, N.H. Matthew

Wong"Evaluating The Visibility of Building Syrian Refugee Shelters by 3D Printing Technology in Jordan"International Journal of Engineering \& Technology-2019
عـدم الإهتمـام بـالتطور التكنولـوجي أثنـاء عمليـة التصــيم والتنفيـذ لمخميات اللاجئين.

\section{IV}

تحقيقاً لأهداف البحث ارتكزت المنهجيـة المتبعـة على محورين رئيسيين

$$
\text { أ- الهنهج النظرى /الوصفى: }
$$

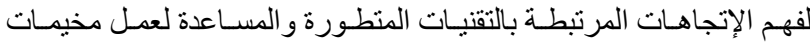
للاجئين، ومدى الإستفادة من هذه التقنيات لتنفيذ المخيمات.

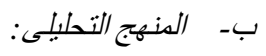

التعرف على مميز ات و إمكانيات التقنيات المساعدة لعمل مخيمات اللاجئين

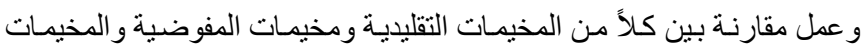
المتطورة وتحليل النتائج وتقييمها لتحديد إيجابيات وسلبيات كلاً من المخيمات.

\section{- V}

يعد تصـيم المخيمات مهمًا حيث أن هذه هي الأمـاكن الأولى التي توفر

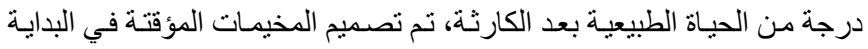
فقط لإحتساب الإحتياجات الحيوية و الوظيفية للضحايا خلال فترة إعادة التوطين.

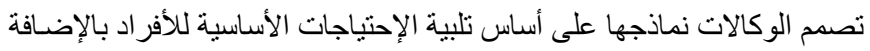

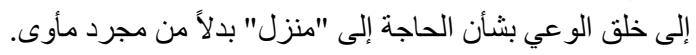

\section{VI}

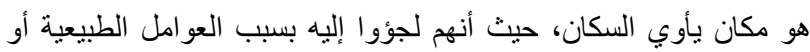

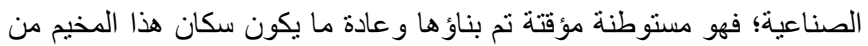

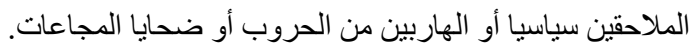

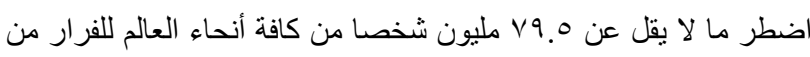

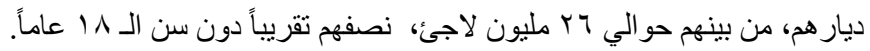

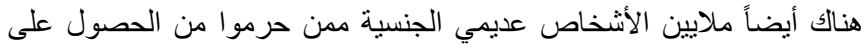

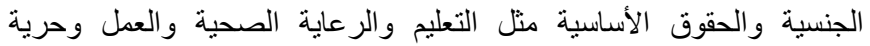

التنقل. (๕)

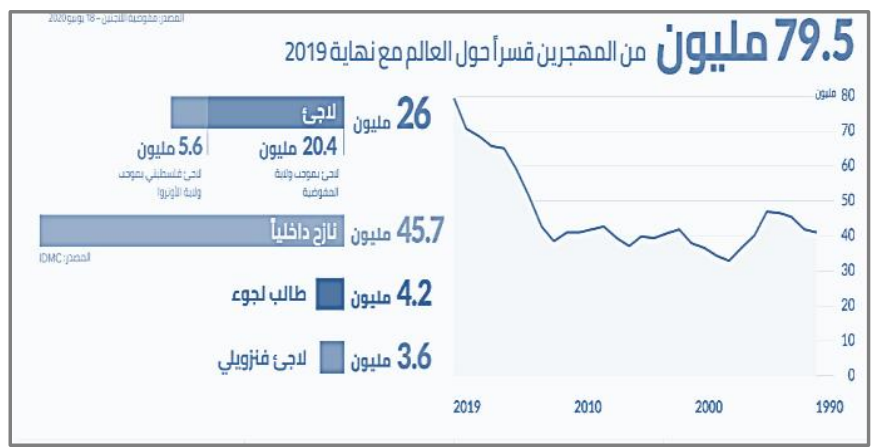

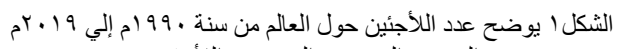
المصدر:المفوضي المصدر:ة اللأجنين

: https://www.unhcr.org/ar/4be7cc27207.html (date18/7/2020 2:53PM) 


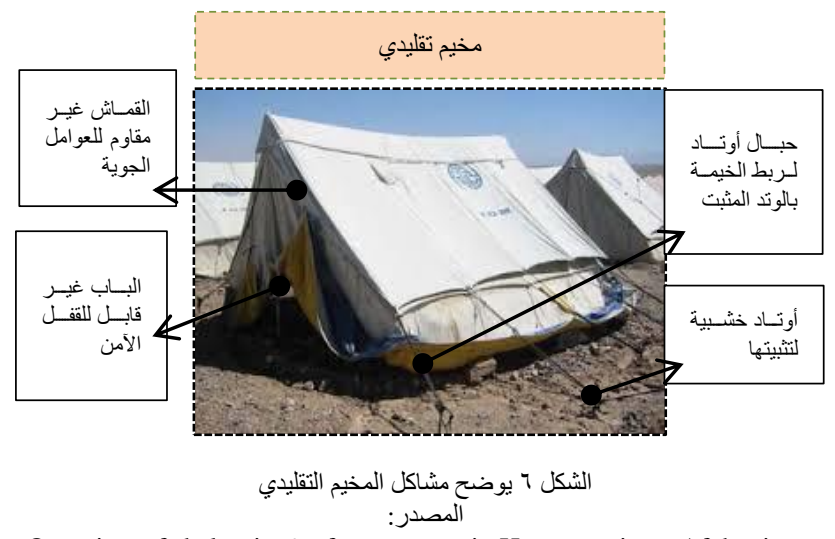

Overview of shelter in 6 refugee camps in Herat province, Afghanistan, March 2002- UNIVERSITY OFCAMBRIDGE- shelterproject.org

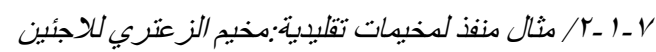

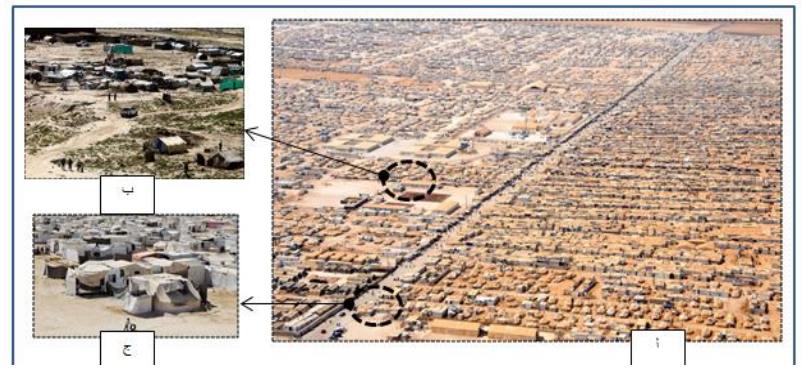

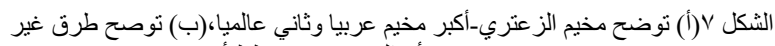

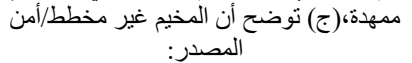

https://jusoorsyria.com/ar/iie-scholarship-in-zaatari/ date3/9/2020 4:53pm

$$
\begin{aligned}
& \text { دراسة تحليلية لمخبمات الزعتري للاجئين بالأردن الارد دن }
\end{aligned}
$$

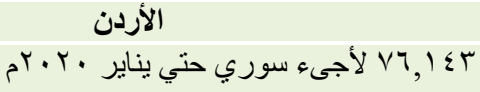

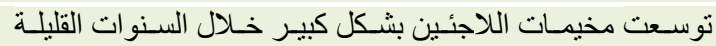

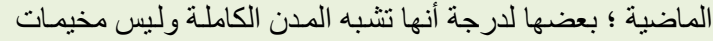
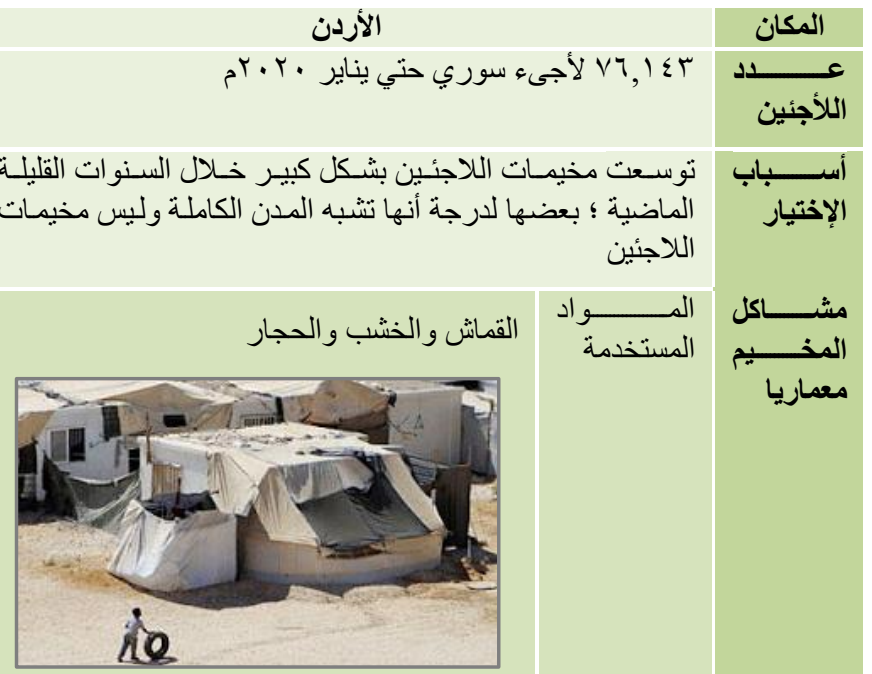

$$
\text { الثكلى يوضح مخيمات الز عتري }
$$

https://jusoorsyria.com/ar/iie-scholarship-in( date3/9/2020 4:53pm)zaatari/

$$
\text { والتبلـة للف لك غير قابلة لأنه صممة بطريقة عشوائية }
$$

\section{.VII}

دخيمات اللاجئين متنو عة فمنها المخيمات التي يقيمها المتضرين (اللاجئين)

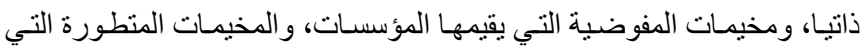
يقيمها المتخصصين ويمكن تصنيفهم إلي ثلاثة أنواع رئيسية:

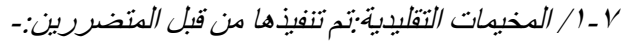

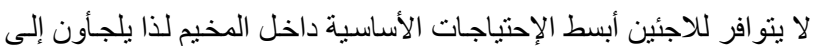

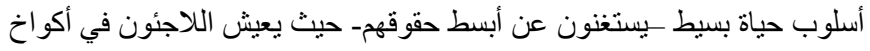

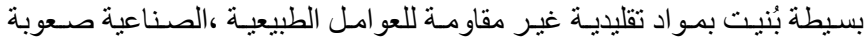

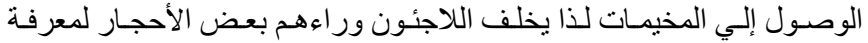

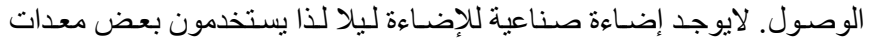

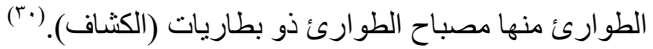

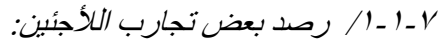

أ-إستخد/م عبوات مبياة بلاستبكية مدلوعة بالرمال.

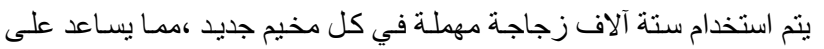

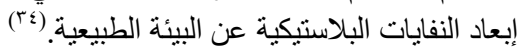

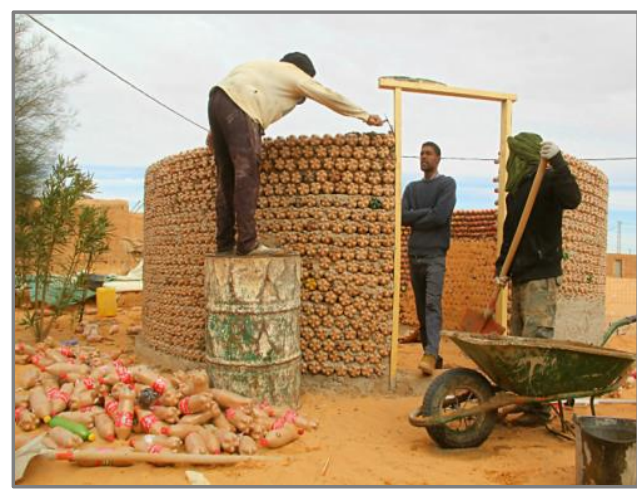

الثكل ؛ يوضح مخيم الزجاجة البلاستيكية بمدينة الزرقاء الأردنية

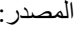

https://earthbound.report/2017/08/18/building-of-the-week-the-plasticbottle-house/ (date4/11/2020 10:05pm)

ب-إستخد/م حاويات السفن" Container ". يتم إستخدام حاويات السفن حل مشاكل سكن اللأجئين من خلال أماكن

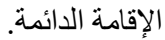

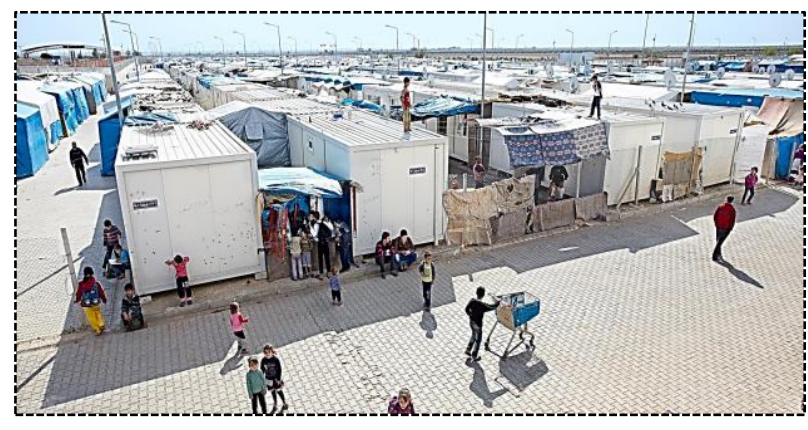

الثكل ه يوضح مخيم للاجئين في تركيا بالقرب من الحدود السورية

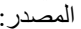

https://www.nytimes.com/2014/02/16/magazine/how-to-build-a-perfectrefugee-camp.html? r=3 (date5/11/2020 10:17am) 


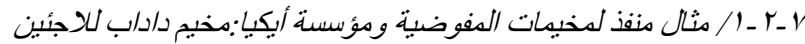

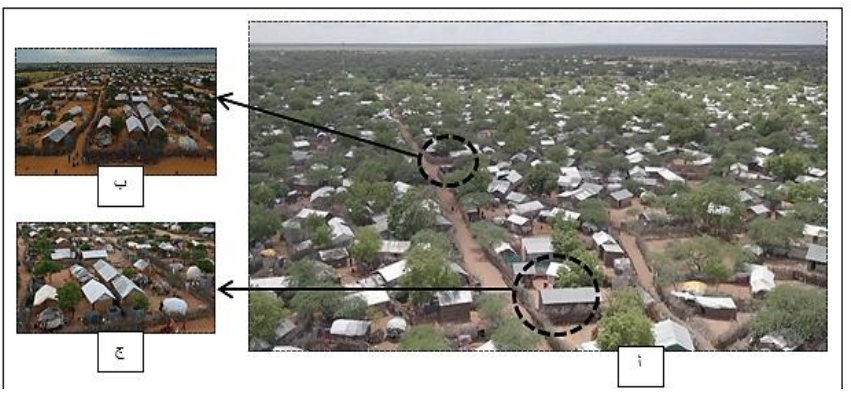

الشكل || (أ) توضح مخيم داداب_من المفوضية، (ب) توضح طرق غير ممهدة، (ج)توضح شكل المخيم المغية

https://www.washingtonpost.com/world/africa/kenya-is-threatening-toclose-the-worlds-largest-refugee-camp/2015/04/27/ab296316-e9cd-11e48581-633c536add4b story.html/ (date 4/9/2020 3:24pm)

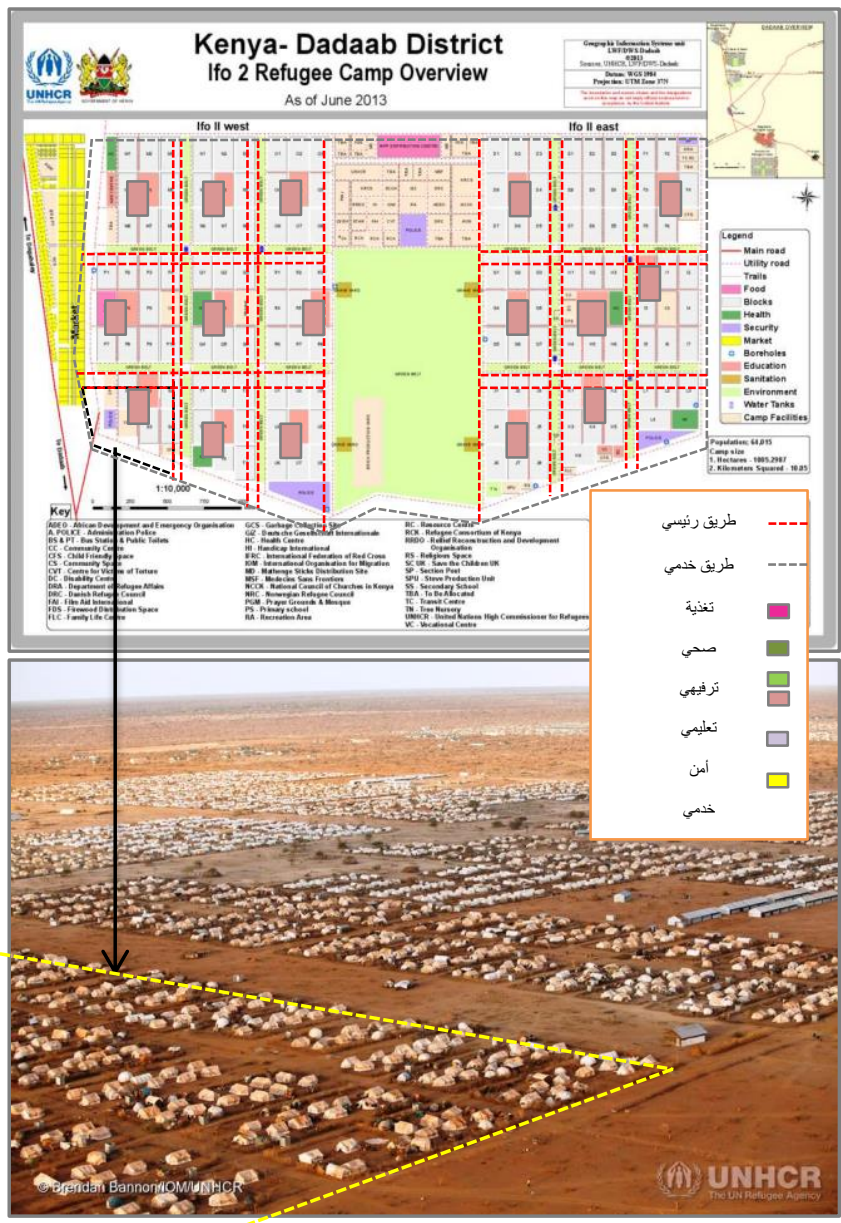

الثكل r ايوضح مسقط أفقي لمخيم داداب من قبل مؤسسة المفوضية

https://data2.unhcr.org/en/documents/details/31898 (date05/11/2020 $12: 19 \mathrm{pm})$

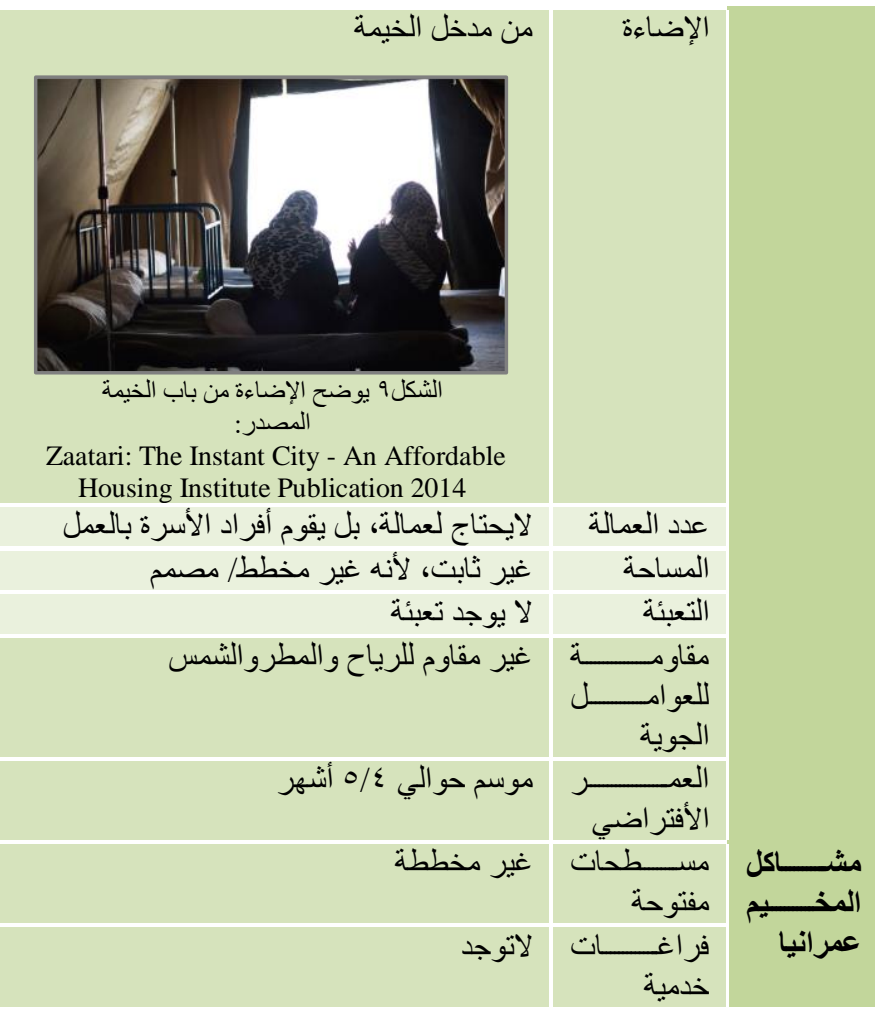

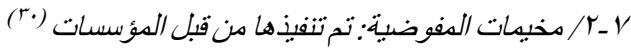

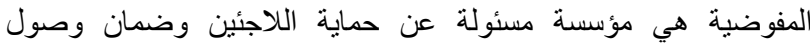

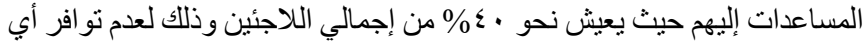

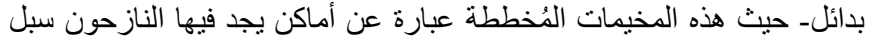

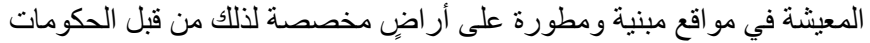

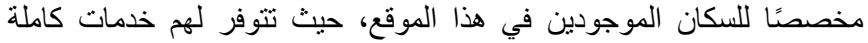

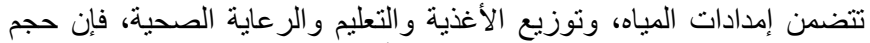

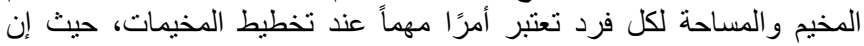

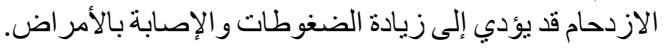

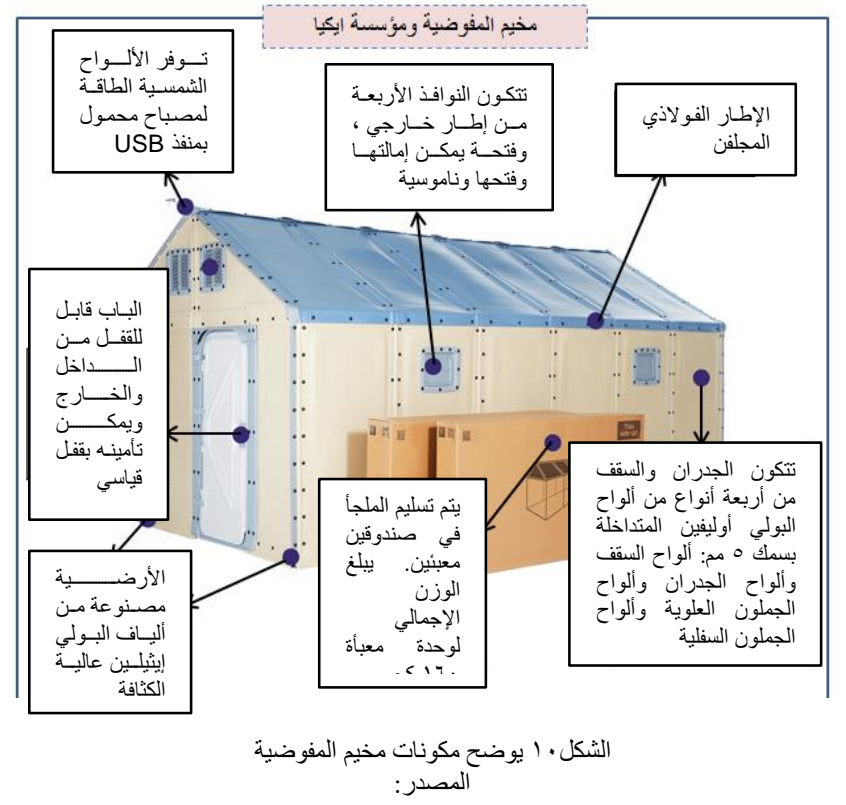

https://bettershelter.org/ (date 18/9/2020 4:23pm) 


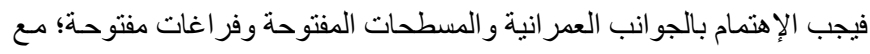

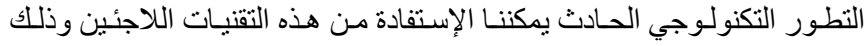

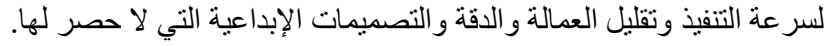

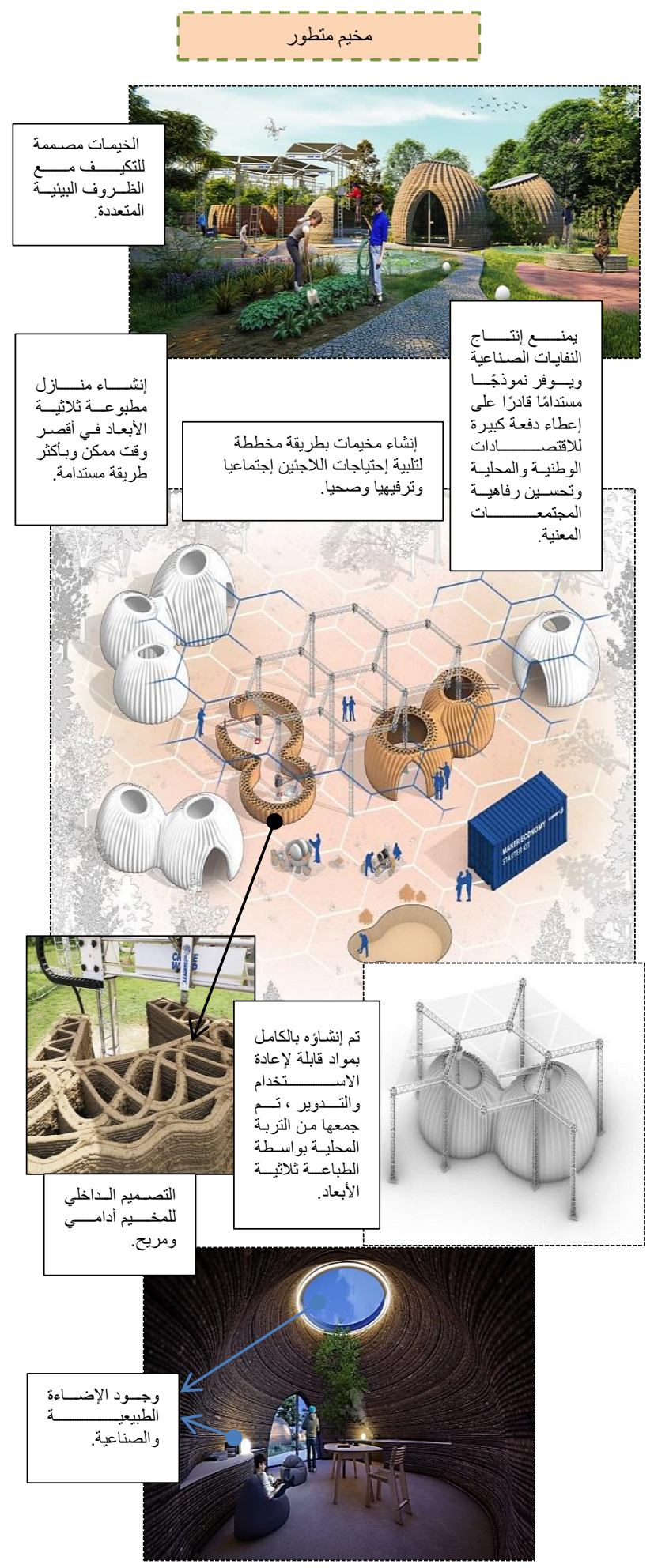

الثكل (14) وضح مميز ات المخيم المتطور

https://www.archdaily.com/927147/mca-and-wasp-design-tecla-a-3d-printedيتصريف الباحثة 10/9/2020 8:20pm)

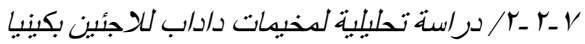

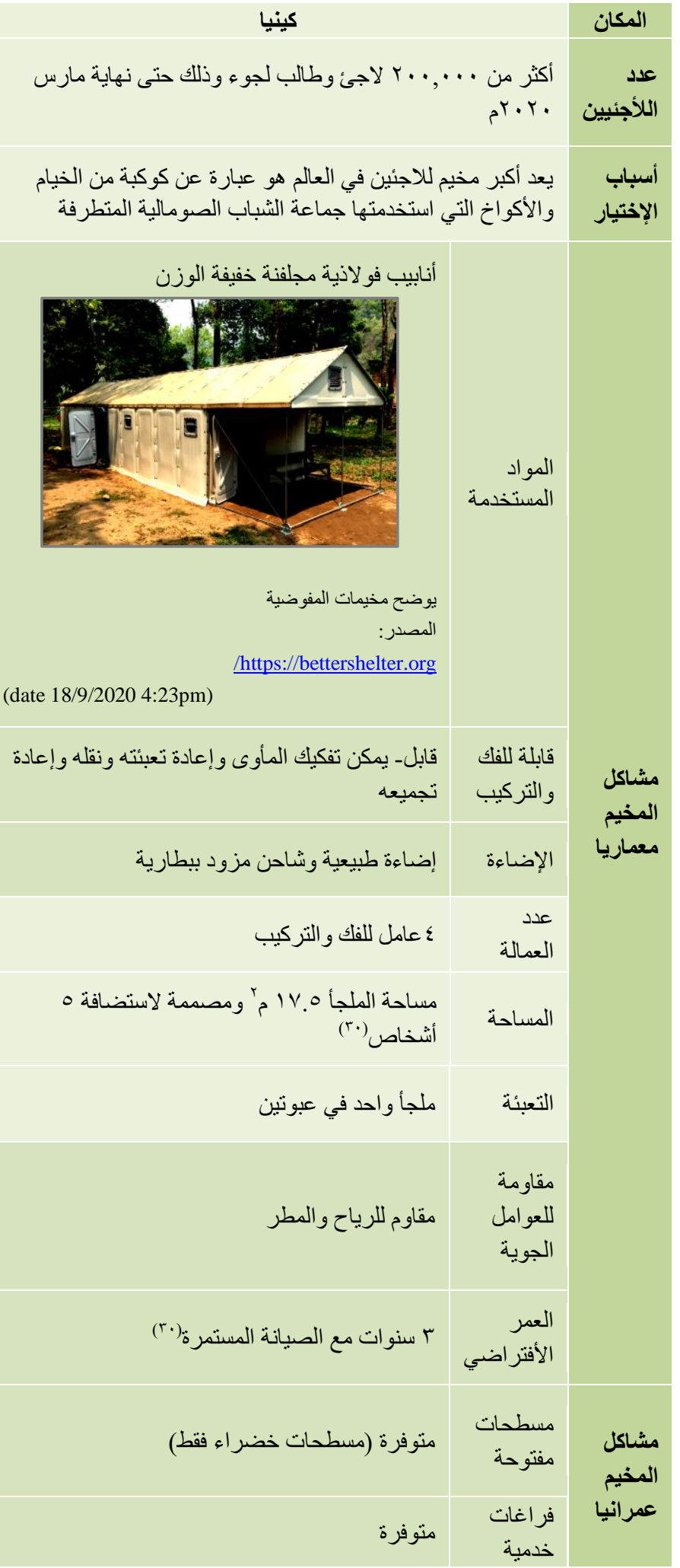

- L / المخبيات المتطورة: تم تنفيذها من قبل المتخصصبن:-

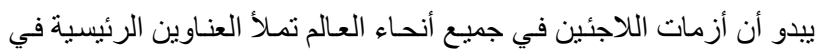

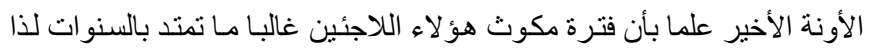




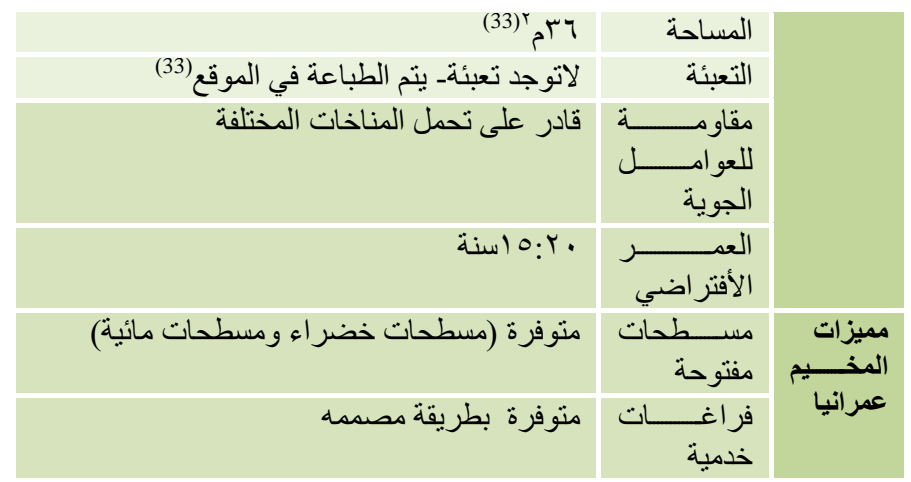

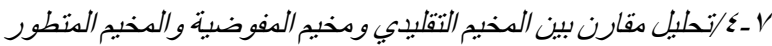

\begin{tabular}{|c|c|c|c|c|}
\hline المخيم المتطور & مخيم المفوضية & المخيم التقلياي & & \\
\hline مو اد محلية تدوير ها- & 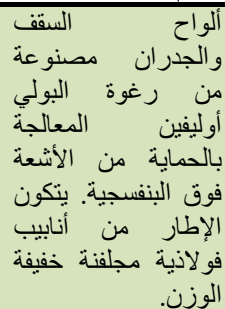 & والقماشر و الخشب & الموتخدمة & \\
\hline التابقة لأنها و وحدات & 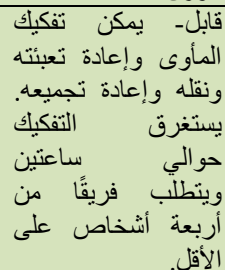 & صشير قابئة بطريقة لأنه & و التركيب للفك & 㩆: \\
\hline إضاءة صناعية طبية & إضـاءة صناعية & من من مدخل الخيمة & الإضـاءة & \\
\hline 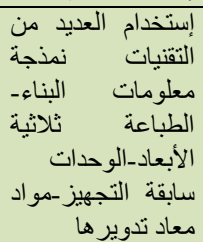 & سابقة التجهيز التقية الوحدات & تلقية بتم استخدام أي & التكنولوجيام & \\
\hline مضوفراء و مسطحات مسطحات & خضرفراء فقط) (مسطحات & غير مخططة & مفتوحة & \\
\hline متوفمة $\quad$ متطريقة & متوفرة & لاتوج & خرمبة & \\
\hline في الموقع المصنع/ في & 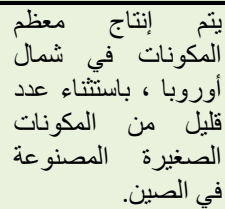 & 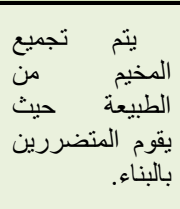 & التصنيع & \\
\hline الأطعابعات و عاملين أُثية & ع عامل & لالإيحتاج ل لعمالة، & عدد العمالة & 司 \\
\hline أفراد الأسرة. للتصيم و عدد & 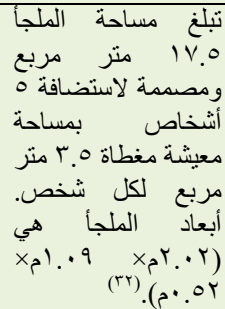 & غير غنابت، لأنه & المساحة & \\
\hline
\end{tabular}

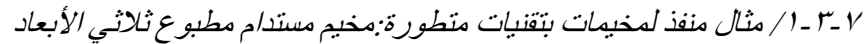

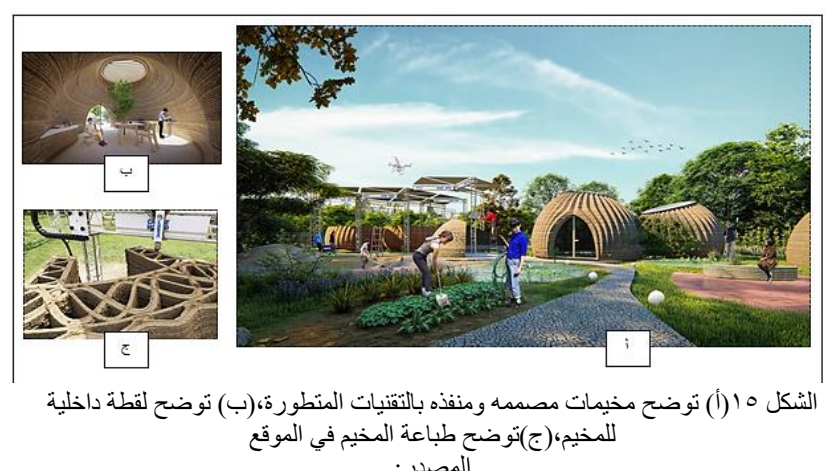

https://www.archdaily.com/927147/mca-and-wasp-design-tecla-a-3dprinted-sustainable-habitat/ (date5/9/2020 4:38pm)

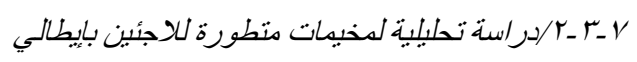
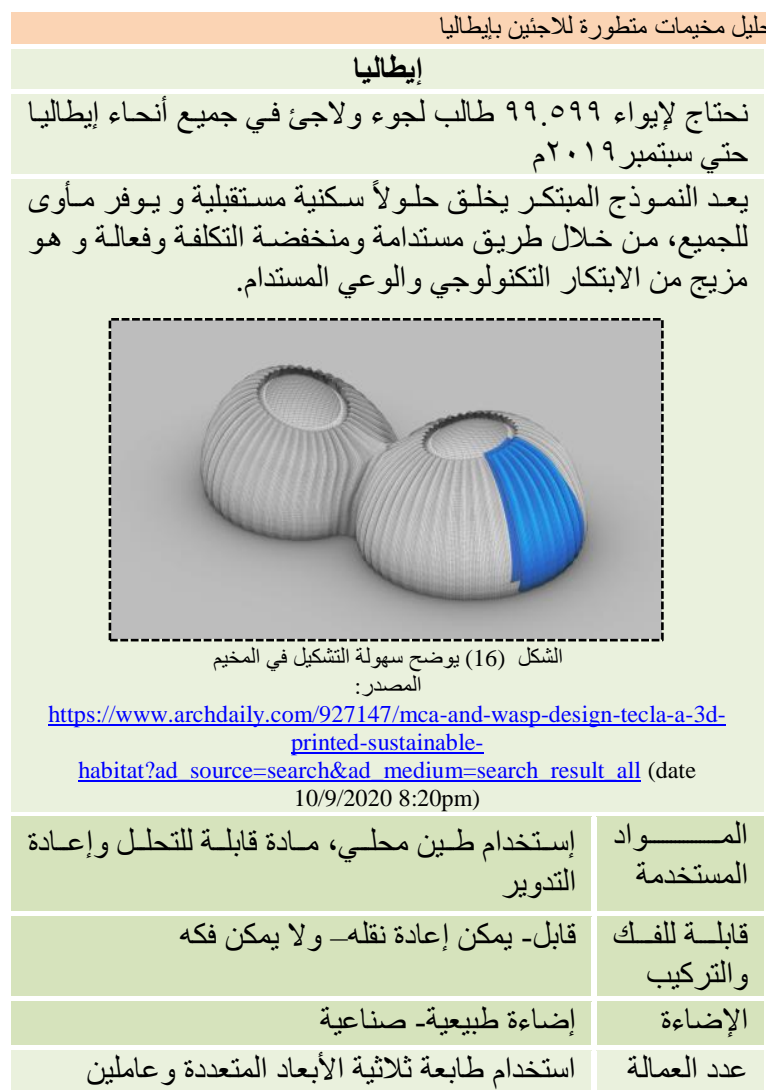

https://www.archdaily.com/927147/mca-and-wasp-design-tecla-a-3dprinted-sustainable-

habitat?ad_source=search\&ad_medium=search_result_all (date 10/9/2020 8:20pm)

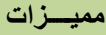

المخــيم

معماريا

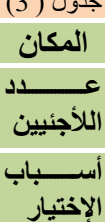

الإختيار

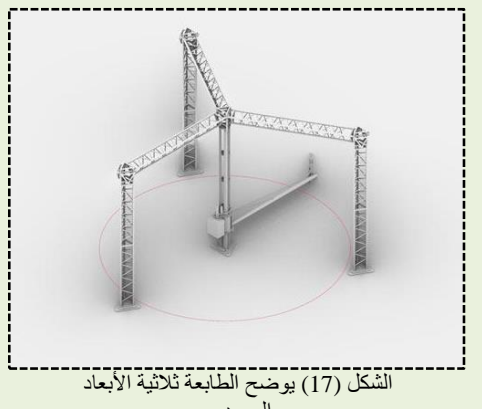

المصدر:

https://www.archdaily.com/927147/mca-and-wasp-

design-tecla-a-3d-printed-sustainable-

habitat?ad_source=search\&ad_medium=search_result all (date 10/9/2020 8:20pm) 


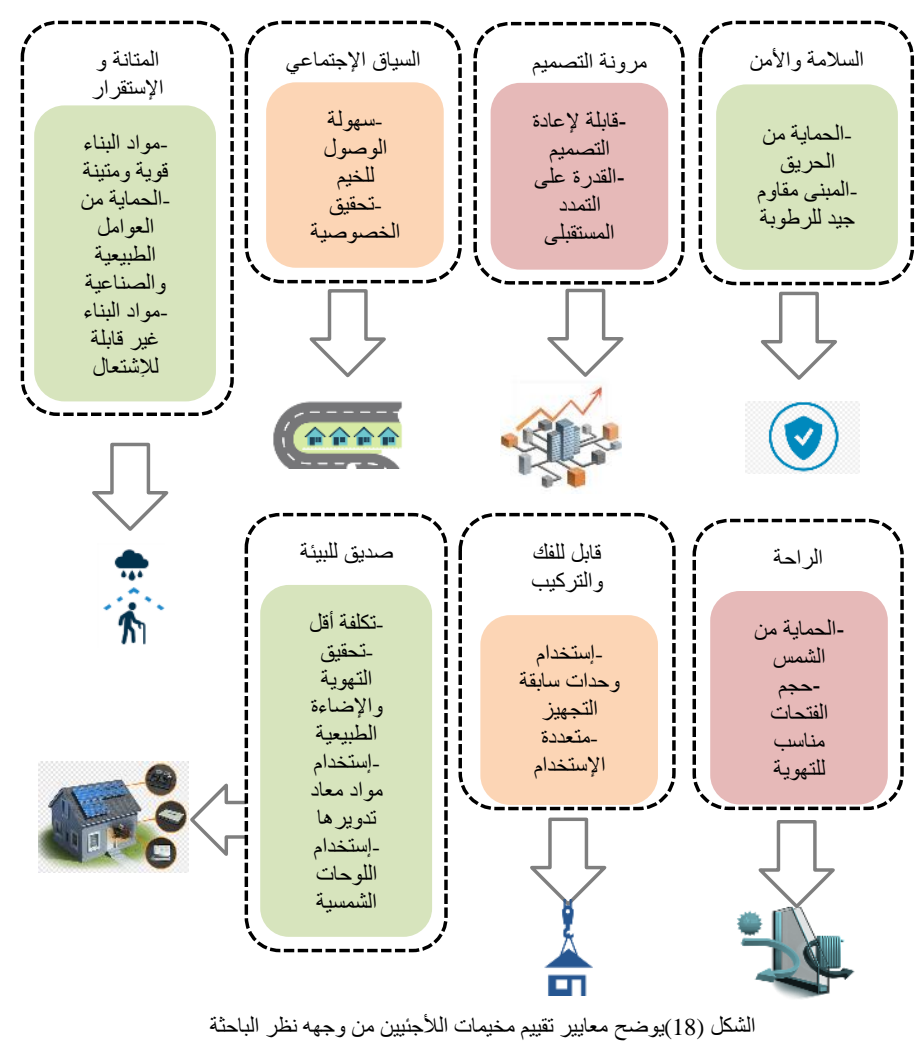

Rania AR. Developing User-Informed Specifications for Refugee Shelters in Hot-Dry Climates: A study of the Al Za'atari Shelter in Jordan. University of Salford; 2017. بتصريف الباحثة

\section{ت تكنولوجيا البناء المتطورة لمخيمات اللاجئين}

يشمل مصطلح "تكنولوجيا البناء المتطورة" مجموعة واسعة من التقنيات

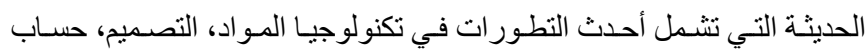

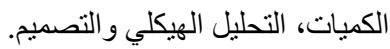

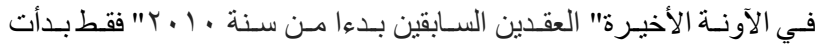

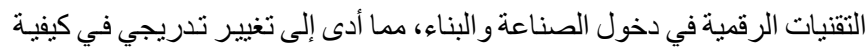

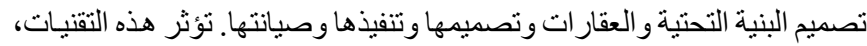

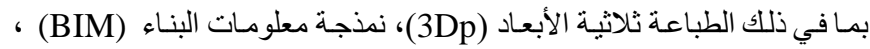

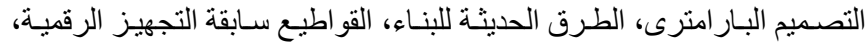

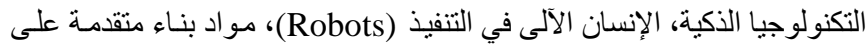

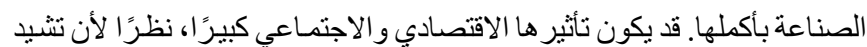

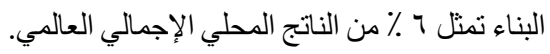

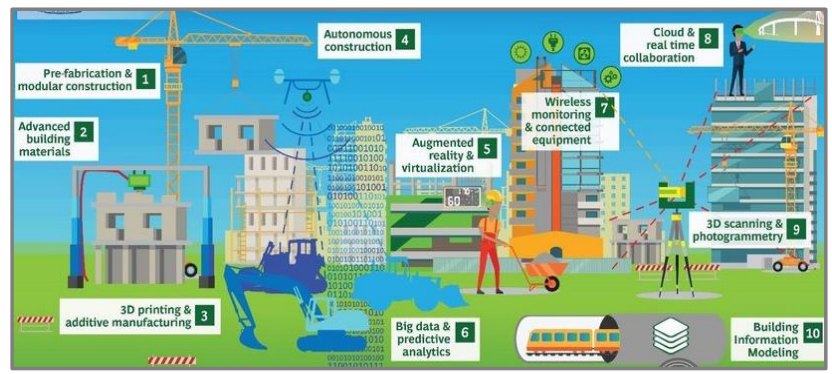

$$
\text { الشكل9 ( يوضح أفضل • ا تقنيات في البناء }
$$

World Economic Forum, Boston Consulting Group 2019

\begin{tabular}{|c|c|c|c|c|}
\hline 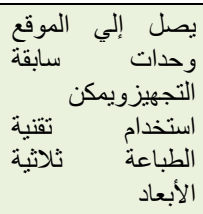 & 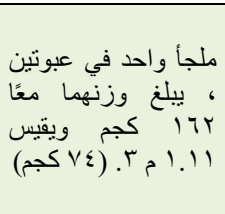 & لا يوجد تعبئة & التعبئة & \\
\hline و والمطرم الثيد للرياح & مقاوم للرياح والمطر & و غيلر مقاوم للرياح & اللعوامل & $\overline{\bar{\sigma}}$ \\
\hline • بــ اسنوات & الصو ثلاث المتوقع اللمأوى مع الأساسية & ع /ه أثثهر & الإفتر اضي & $\because 0$ \\
\hline
\end{tabular}

الجدول ايوضح تحليل مقارن المخيم التقليدي ومخيم المفوضية والمخيم المنطور

\section{.VIII}

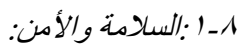

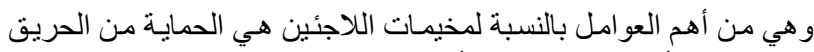
لسهولة اشتعالها وأيضا الحماية من الأمطار و الرطوبة المبة

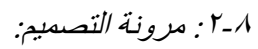

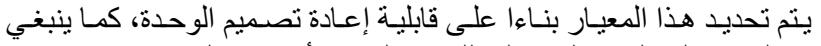
مر اعاه القدرة على التمدد المستقبلي للوحدة الو احدة أومجمع الوحدات.

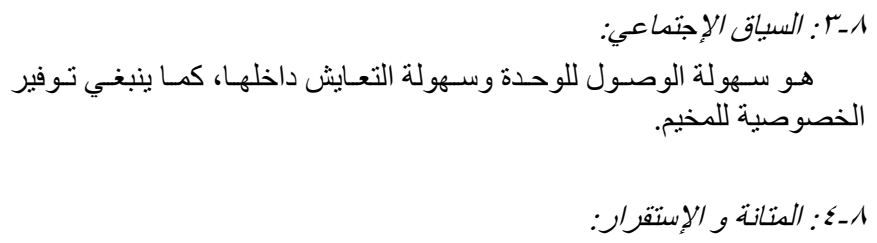

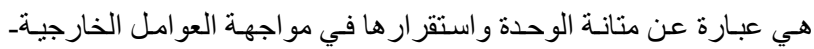

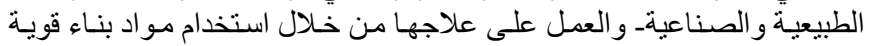
ومتينة غير قابلة للإنشتعال و تتحمل هذه العو امل.

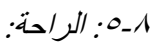
يجب تحقيق الر احة الحر ارية سو اء من الثمس و الحرارة والرطوبـة أو من خلال التهوية الطبيعية للمخيم.

1ـ ـ : قابل للفك والتركبي:

يجب أن تكون الوحدات/المخيمات قابلة للإستخدامات المتعددة يمكن فكها وتركيبها بطرق مختلفة لإعطاء حلول أكثر.

يمكن إستخدام مو اد بناء معاد تدوير ها أو إستخدام مو اد بيئية مستدامة محلية

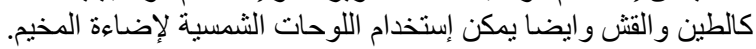


يعد الجهاز أداة موسيقية بحاجة إلى تعلم العزف عليهاء

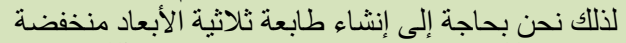

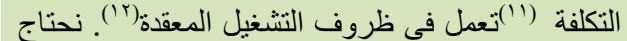

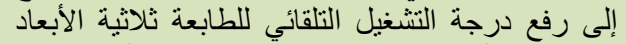

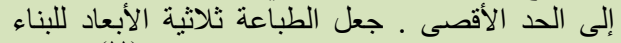

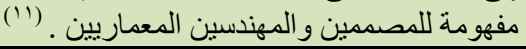

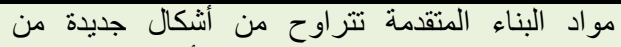

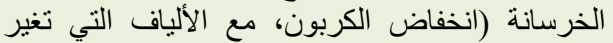

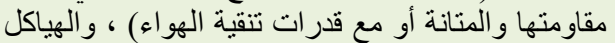

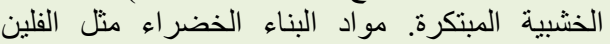

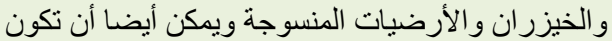
جز عا من مواد البناء المتقدمة.

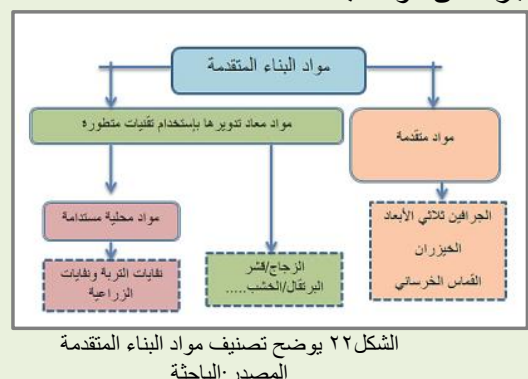

عندما يتم تكديس أوراق الجرافين بدقة فوق بافئ بعضها

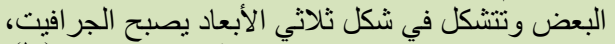

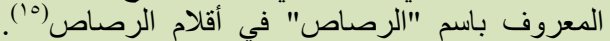

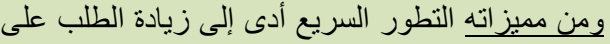

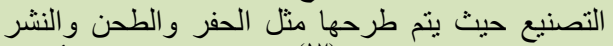

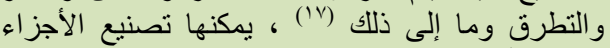
ثلاثية الأبعاد مباشرةً من خلاد إلى تكديس الطبقة المتسلسل

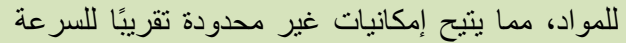

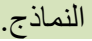

يستخدم الخيزران كمواد بناء بسبب قيمته الجمالية القوية

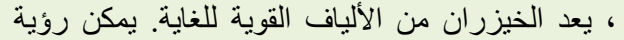

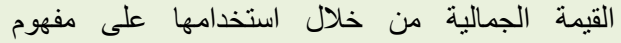

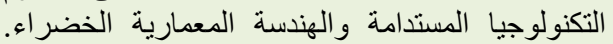

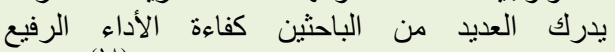

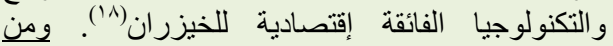

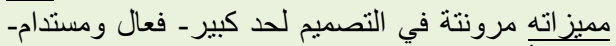
الحد الأدنى من تكلفة البناء وسهل التهل الاستخدام- قوة الثدي:

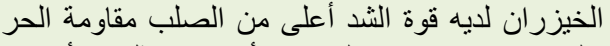

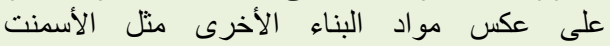
والأسبستوس ، لا يشكل الخيزران أي خطر على على الأ عن

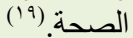

هو قماش مرن ثلاثي الأبعاد مشبّع بالأسمنت؛ يتصلب

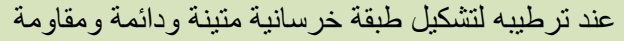

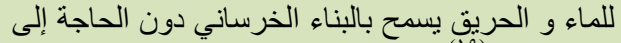

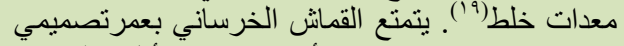

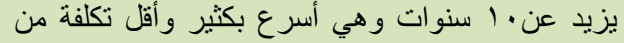

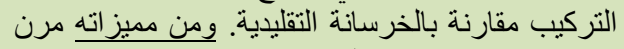

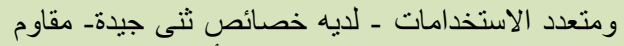

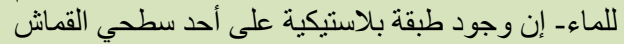

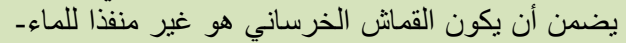

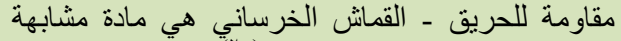

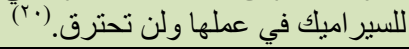

9 ـ ا/إستعر/ض بعض التقنيات الحديثة التـي تساعد المصدمين في تصميج وتنفيذ

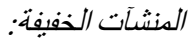

د/التحديات

أ/مقدمة

أولا:

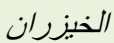

ثالثا :

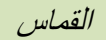

الخرساني
يعرضرض البحـث مجمو عـة مـن التقنيـات الحديثة التي تم تطبيقهـا في العقد

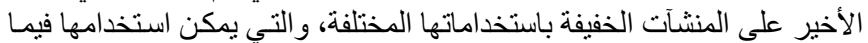
بعد لتطوير اشكال و تصميم ملاجئ اللاجئين.

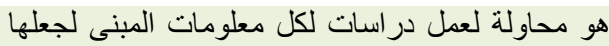

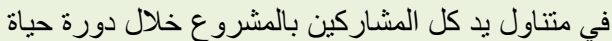

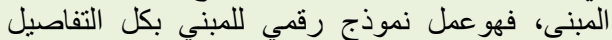

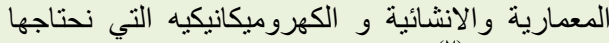

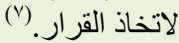

هي أداة ضبط للمشروع قبل تتفيذه فى الو اقع، كما تساعد

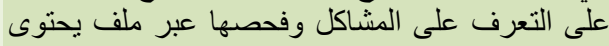

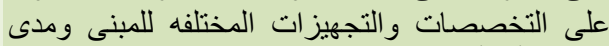

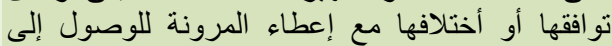

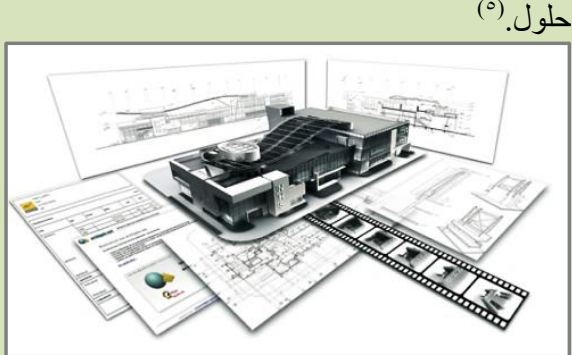

الثكل • Y بوضح الفكرة العامة لنمذجة معلومات البناء

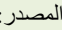

http://www.buildersdepot.biz/en/training/training/ (date6/9/2020 9.00pm)

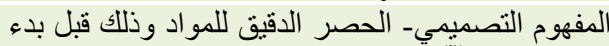

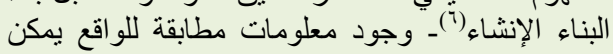

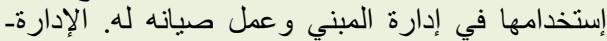

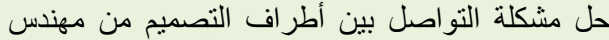

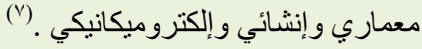

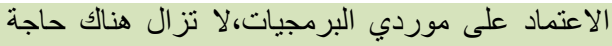

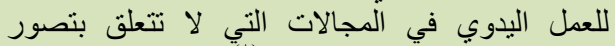

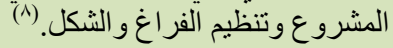

يشار إليها أيضًا باسم الطباعة المضافة أو التصنيع الإضافي Additive Manufacturing -AM هي

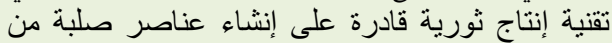
ملف رقمي تم تحميله إلى طابعة ثلاثية الثاثة الأبعاد.

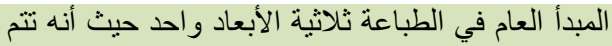
عملية الطباعة من خلال رص طب طبقات المبات المادة (الخامة)

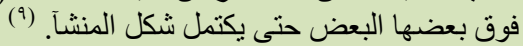

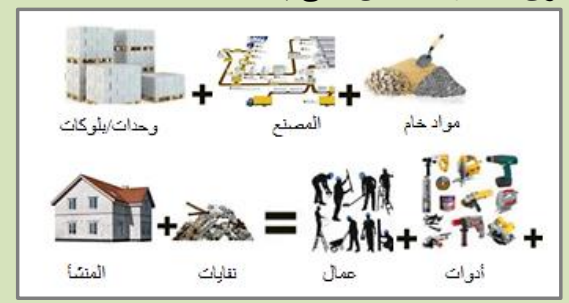

الشكل اب يوضح الفكرة العامة للبناء التقلبدي و البناء بالطباعة ثلاثبة الأبعاد https://medium.com/@Nik chen/what-is-construction-3dprinting-perspectives-and-challanges-5b57170c2a29 (date29/1/2018 9.00pm)

تكاليف أقلـ- البناء المستدام/صديق للبيئة- ـالبناء في البي

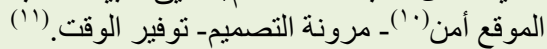

د/التحديات

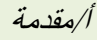

ب/الفكرة

العامة الفكرة

ج/المديزات 
تخزين أكثر ملائمة يؤدي إلى الحد الأدنى من حالات

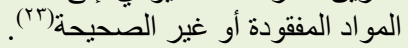

يجب تحديد خطة النقل مع الطرق و التوقيت لتوفير الأمان إندان

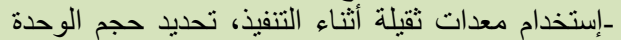

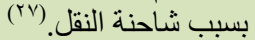

د/التحديات

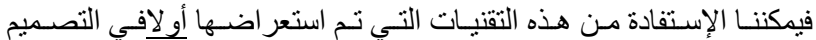

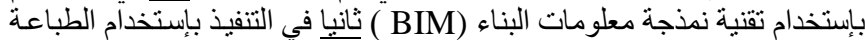

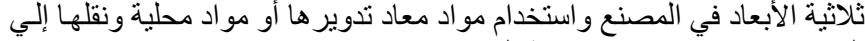
الموقع في صورة وحدات سابقة التجهيز.

\section{X}

تهدف الورقة البحثية إلي إظهار فائدة استخدام بعض التقنيات المتطورة مثل التيل

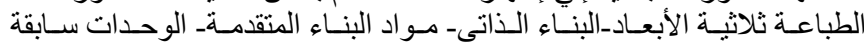

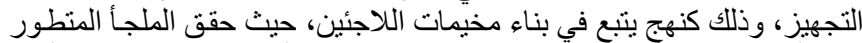

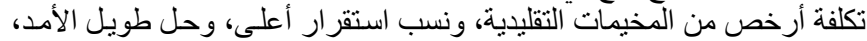

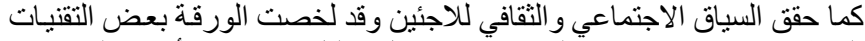

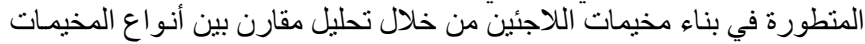

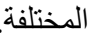

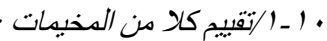

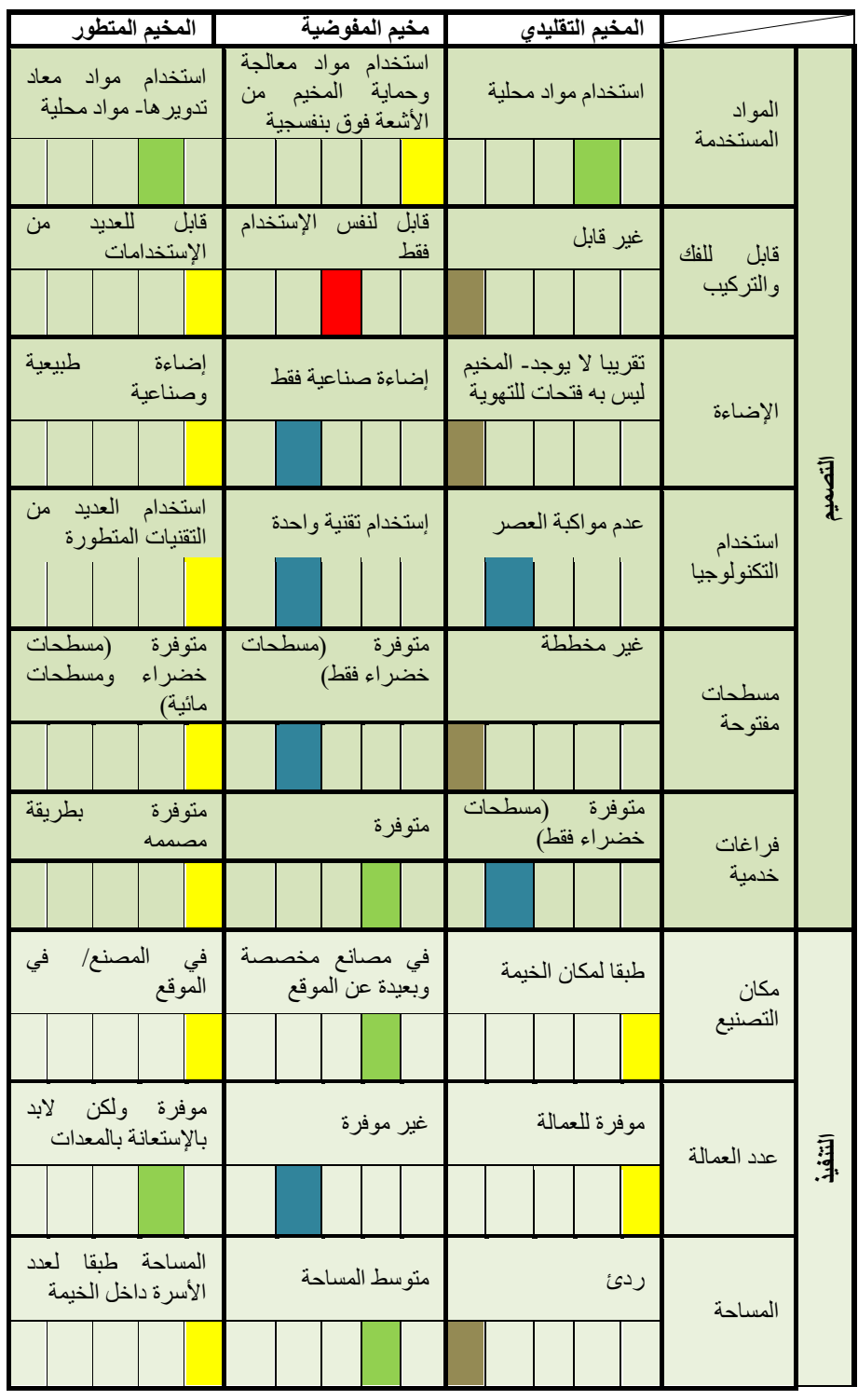

يمكن اعتبار الطباعة ثلاثية الأبعاد تقنية تصنيع موزعة

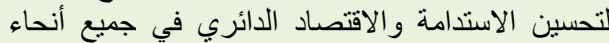

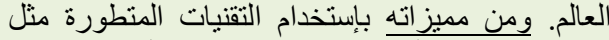

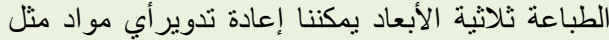

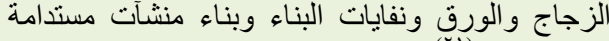

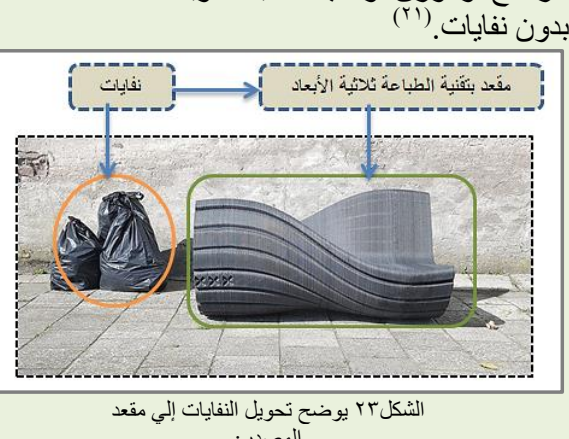

inhabitat This brilliant project turns plastic waste into 3Dprinted benches for Amsterdam (date10/9/2020 7.52pm)

يمكن إستخدام نفايات التربة والزراعة إعة لعمل منشاً

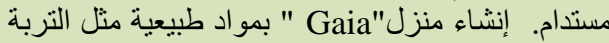

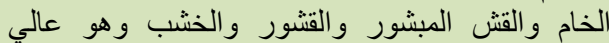

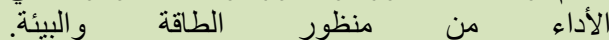

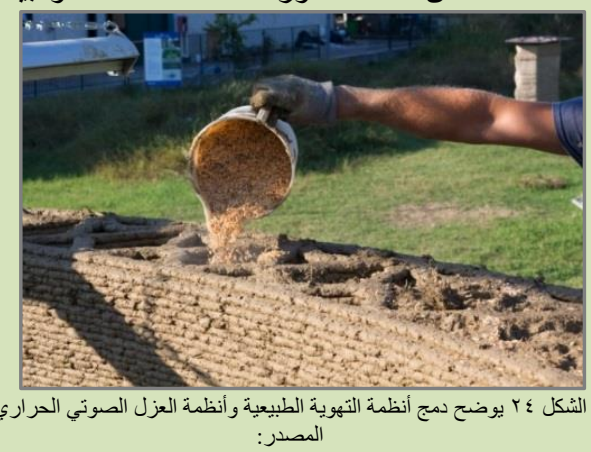

https://www.3dwasp.com/stampante-3d-per-case-crane-wasp/ (date16/3/2020 3.00pm)

تعتبر التجهيز المسبق إحدى الإستراتيجيات التي يتم

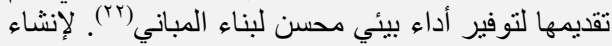

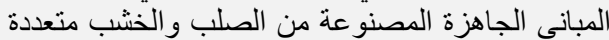
الوحدات السكنية إن هذا النوع من البناء يوفر الدئ أداء بيئًا

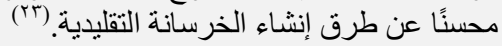
المباني الجاهزة هي إنثاء ألو اح ووحدات مسبقة الصنع أنساء

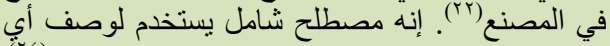

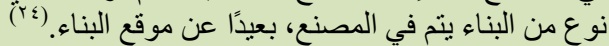

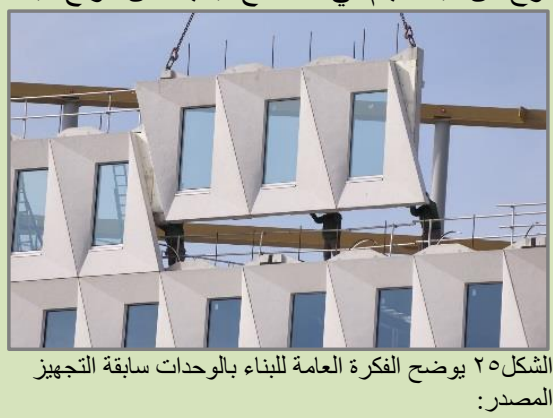

https://www.saint-gobain.com/en/prefab-revivalconstruction (date10/9/2020 8:03pm)

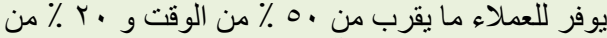

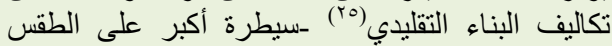

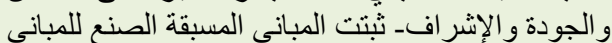

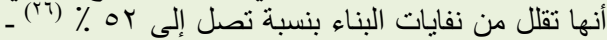

ب/مو/د

معاد

تدوبيرها

بإبتخدام

تقنيات

متطورة

ج/مواد

محلية

مستدامة

أرمقدمة 


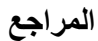

[1] Alshawawreh L, Smith RS, Wood JB. Assessing the sheltering response in the Middle East: Studying Syrian camps in Jordan.IntJ.Humanit.SocSci Availablefrom: https://waset.org/publications/10007596/assessing-the-shelteringresponse-in-the-middle-east-studying-syrian-camps-in-jordan. (date 16/9/2020 10:49pm)

[2] SyndiGate.info. Halabat: the first ever images from the new Syrian refugee camp in Jordan | Al Bawaba Available from: https://www.albawaba.com/editorchoice/new-zaatari-refugee-camp466705 (date23/4/2018 4:05pm)

[3] Mohanad Akeila, Kelvin Kuok King Kuok, N.H. Matthew Wong"Evaluating The Visibility of Building Syrian Refugee Shelters by 3D Printing Technology in Jordan"2019-International Journal of Engineering \& Technology.

[4] https://www.unhcr.org/ar/4be7cc27207.html (date 16/9/2020 10:51pm)

[5] http://www.buildersdepot.biz/en/training/training/ (date 6/9/2020 $9.00 \mathrm{pm})$

[6] Yusuf arayici -s.coates-lauri j.koskela-mike kagioglou"BIM implementation for architectural practice"2011-ResearchGate.

[7] www.autodesk.com/top 10 benfits of BIM. (date 18/8/2020 9:05pm).

[8] Olga Gamayunova-Nikolai Vatin"BIM-Technology in Architectural Design"2014-researchgate.

[9] http://ar3dprinter.com/3dprinting/ (date 23/11/2019 9:45pm)

[10] https://www.whatsnextcw.com/5-ways-3d-printing-transformingconstruction/-Technology \& Innovation - (date 12/02/2018 5:45pm)

[11] Mehmet Sakin, Yusuf Caner Kiroglu"3D Printing of Buildings: Construction of the Sustainable Houses of the Future by BIM"-2017 ScienceDirect.

[12] https://medium.com/@Nik_chen/what-is-construction-3d-printingperspectives-and-challanges-5b57170c2a29 (date 29/01/2018 9:48pm)

[13] https://www.robotshop.com/community/blog/show/how-robots-arerevolutionizing-the-construction-of-houses-and-buildings (date 6/12/2018 9:48pm)

[14] Robotics Online How Construction Robots Are Disrupting The Industry By Robotics Available from: https://www.robotics.org/blog-article.cfm/How-Construction-Robotsare-Disrupting-the-Industry/90 (date 16/9/2020 11:08pm)

[15] https://www.bigrentz.com/blog/autonomous-construction-vehicles (date 29/12/2019 10:00pm)

[16] Twenty-Two Architectural Magazian- Architecture and Technology 2016.

[17] Nestor Washington Solís Pinargote, Anton Smirnov ,Nikita Peretyagin,Anton Seleznev and Pavel Peretyagin" Direct Ink Writing Technology (3D Printing) of Graphene-Based Ceramic Nanocomposites: A Review"2020- nanomaterials Mdpi. Available from: https://www.mdpi.com/2079-4991/10/7/1300/htm (date 16/09/2020 11:18pm)

[18] Zafuan Husri,Mohd Sabrizaa,Suzana Said,Razali Kamisan,Nordin Misnat,Rahim Ismail,Mohd Tarmizi-"Bamboo Modular System (BMS) for New Eco Architeture" Conference Paper • January 2014ResearchGate

[19] https://theconstructor.org/building/bamboo-as-a-building-materialuses-advantages/14838/ date 31/1/2020.

[20] Qasim M. Shakir"concrete cloth" July 2019-University Of Kufaresearchgate.

[21] https://theconstructor.org/concrete/concrete-cloth-propertiesapplications-process/17070/ (date 20/1/2020 1:30pm)

[22] Jukka Pakkanen, Diego Manfredi, Paolo Minetola, and Luca Iuliano" About the Use of Recycled or Biodegradable Filamentsfor Sustainability of 3D PrintingState of the Art and Research Opportunities" 2017- researchgate.

[23] LU Aye,T.Ngo,R.H.Crawford,R.Gammamplia,P.Mendis." Life cycle greenhouse gas emissions and energy analysis of prefabricated reusable building modules"2012 Energy and Buildings.

[24] Tharaka Gunawardena, Priyan Mendis, Tuan Duc Ngo" Performance of multi-storey prefabricated modular buildings with infill concrete walls subjected to earthquake loads" research paper published on September 2017- researchgate.

[25] Tharaka Gunawardena, Priyan Mendis ,Tuan Duc Ngo ,Lu Aye " Sustainable Prefabricated Modular Buildings"December 2014researchgate.

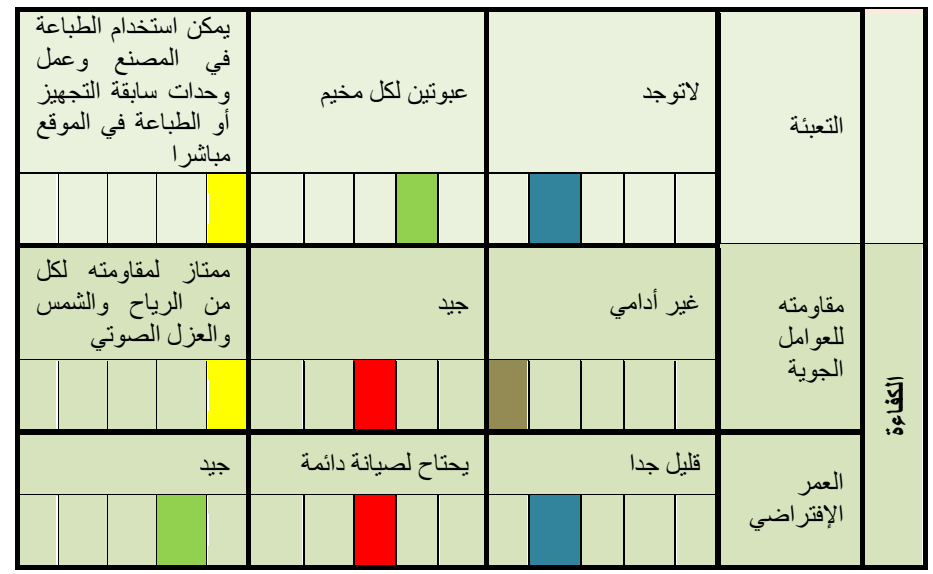

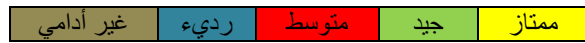

$$
\begin{aligned}
& \text { الجدول : يوضح تقييم كلا من المخيم التقليدي ومخيم المفوضية و المخيم المتطور } \\
& \text { XI }
\end{aligned}
$$

حيث أن موضوع تكنولوجيا البناء وتأثثير ها على العمارة بشكل عام المات و الفكر

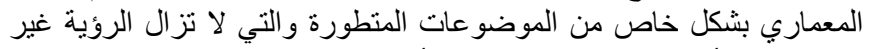

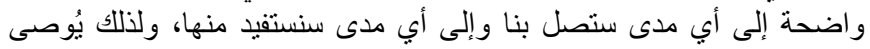

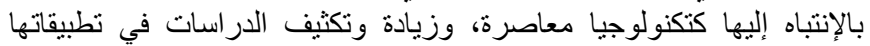
و امكانياتها.

• ورورة عمل دراسات مكثفة على إستخدام التقنيات المنطورة التي تم

ذكر ها.

• وعي المجتمع بإستخدام المواد المعاد تدويرها وتصنيفها لسهولة

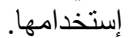

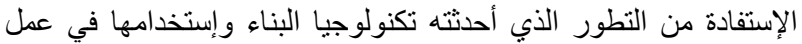
منشآت خفيفة وسريعة و أدامية وقابلة للفك و التركية التركيب.

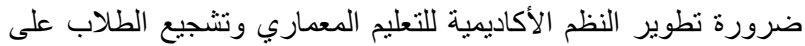

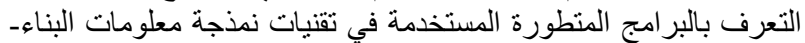

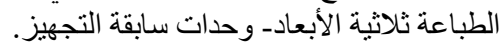

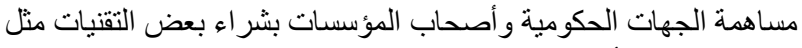
الطباعة ثناثية الأبعاد و غير ها.

$$
\begin{aligned}
& \text { مساهمة الباحثين } \\
& \text { قامت المؤلفة م/أسماء الطويل بعدل الآتي: } \\
& \text { - - إقتر اح فكرة البحث. - } \\
& \text { - - جمع البيانات وتحليلها. } \\
& \text { - عمل المنهجية. } \\
& \text { - - تصميم وتنسيق البحث. - }
\end{aligned}
$$

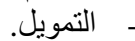

$$
\begin{aligned}
& \text { - المر اجعة النقدية من قبل المحكمين. } \\
& \text { حيث قام المؤلف د/مدحت سمرة بعمل الآتي: }
\end{aligned}
$$

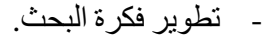

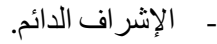

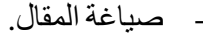

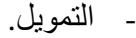

أوجة الثكر إلي أ.م.د/أحمد الطنطاوي- رئيس قسم الهندسة المعمارية

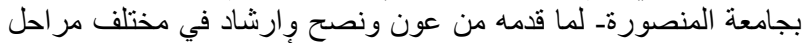

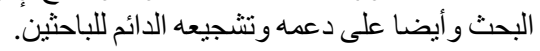


Title in Arabic:

$$
\text { تأثير تكنولوجيا البناء المتطورة على مخيمات اللاجئين }
$$

\section{Abstract in Arabic:}

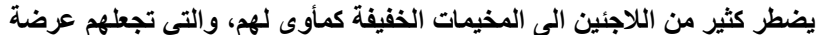

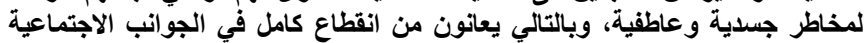

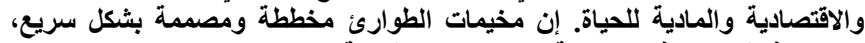

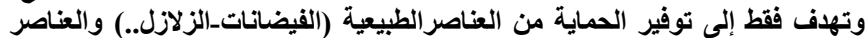

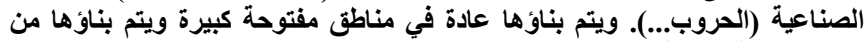

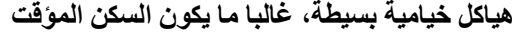

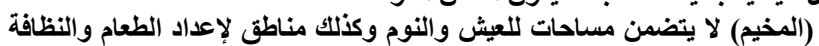

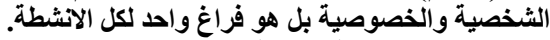

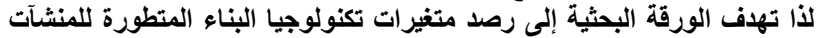

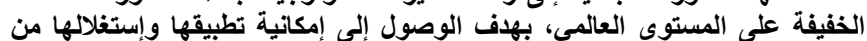

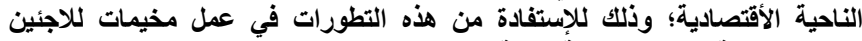

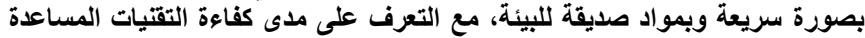

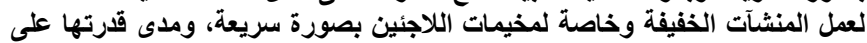
التعامل مع عمليات إبداعية معقدة.

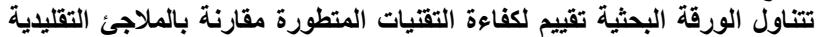

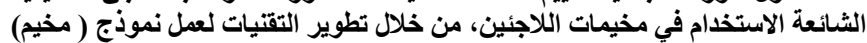

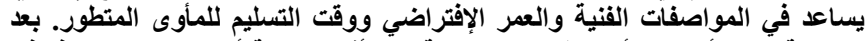

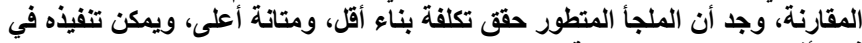
فترة أقل من الملاجئ التقليدية.
[26] Jaillon, L., Poon, C.S. and Chiang, Y.H "Quantifying the Waste Reduction Potential of Using Prefabrication in Building Construction in Hong Kong"- 2009- Waste Management.

[27] Andriel Evandro Fenner, Charles Joseph Kibert and Victoria Zoloedova" Conference Report 2017: State-of-the-art of Modular Construction" researchgate publish in October 2017.

[28] https://www.unhcr.org/7steps/ar/shelter/- (date16/9/2020 11.22pm)

[29] The UNHCR Global Strategy on Settlement and Shelter 2014-2018 Available from:http://www.unhcr.org/shelterstrategy. (date 16/9/2020 $11: 24 \mathrm{pm})$

[30] SHELTER-DESIGN-CATALOGUE-January2016-Availablefrom: https://cms.emergency.unhcr.org/documents/11982/57181/Shelter+Des ign+Catalogue+January+2016/a891fdb2-4ef9-42d9-bf0fc12002b3652e (date5/9/2020 3:58pm)

[31] https://bettershelter.org/ (date 18/9/2020 4:23pm)

[32] https://www.archdaily.com/927147/mca-and-wasp-design-tecla-a-3dprinted-sustainable-

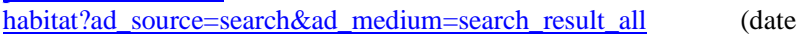
10/9/2020 8:20pm)

[33] https://earthbound.report/2017/08/18/building-of-the-week-the-plasticbottle-house/ (date 4/11/2020 10:05pm) 